Pacific Journal of Mathematics

SOME MINIMUM PROBLEMS IN THE THEORY O 


\title{
SOME MINIMUM PROBLEMS IN THE THEORY OF FUNCTIONS
}

\author{
D. R. SCHOLZ
}

1. Introduction. This paper is concerned with extremal problems of the type

$$
\begin{aligned}
& \iint_{D}\left|f^{\prime}(z)\right|^{2} d x d y=\lambda=\text { minirium: } \\
& \iint_{D} \sigma(z)|f(z)|^{2} d x d y=1,
\end{aligned}
$$$$
\sigma(z)>0
$$

where $f(z)$ belongs to the class of analytic functions which are regular and single-valued in a given domain $D$. A further slight restriction of this class is necessary in order to exclude the trivial solution $f(z)=$ constant.

While this problem has sufficient formal similarity to the classical eigenvalue problems of mathematical physics to make some of the classical results applicable, it will be shown that it leads, on the other hand, to reproducing kernels of a type analogous to those considered by Bergman [1]. With certain peculiar restrictions it will be shown also that the solution of this problem is, up to constants, the resolvent kernel $K(z, w ; \lambda)$ of an integral equation

$$
f(w)=\lambda \iint_{D} \overline{K(z, w, a)} f(z) d x d y
$$

whose solution is identical with that of (1.1). The kernel $K(z, w, a)$ of (1.2), and hence both $K(z, w ; \lambda)$ and the solution of (1.1), are related to certain canonical conformal mapping functions.

2. The eigenvalue problem. I et $F(z)$ be analytic in a given finite, multiply connected domain $D$. Among all analytic, single-valued functions, let $f_{1}(z)$ be the function minimizing the area integral

Received June 9, 1953. The author wishes to express gratitude to Professor Zeev Nehari of Washington University for his direction in the preparation of the author's doctoral dissertation, the principal contents of which are represented in this paper.

Pacific J. Math. 4 (1954), 275-299 


$$
J_{D}(f)=\iint_{D}\left|f^{\prime}(z)\right|^{2} d x d y
$$

under the conditions

$$
H_{D}(f)=\iint_{D} \sigma(z)|f(z)|^{2} d x d y=1, \quad \sigma(z)=|F(z)|^{2},
$$

and either

$$
f(a)=0, \quad a \in D
$$

or

$$
\iint_{D} \sigma(z) f(z) d x d y=0
$$

and let $\lambda_{1}$ be the minimum value of (2.1). Conditions of the type (2.3) or (2.4) are necessary in order to exclude the trivial solution $f(z)=\alpha=$ const., $\alpha \neq 0$. We denote by $\lambda_{n}$ the successive minima of (2.1) under the additional conditions

$$
\iint_{D} \sigma(z) f(z) \overline{f_{k}(z)} d x d y=0
$$

$$
(k=1,2, \cdots, n-1),
$$

where $f_{k}(z)$ denotes the $k$ th eigenfunction.

The subsequent results will be stated principally for that class of functions, denoted by $L^{2}(a, D)$, for which (2.1) exists and (2.3) holds. In each situation, however, one obtains a similar and generally somewhat simpler analysis and result for the class of functions $L^{2}(D)$ satisfying (2.4).

Our eigenvalue problem presents many formal analogies to the classical eigenvalue problems of mathematical physics [4]. There is, however, an essential difference between the two types of problems. While in the classical case the class of functions competing in the minimum problem is very general and is only restricted by certain homogeneous boundary conditions, we restrict our attention at the outset to the class of analytic functions which are regular and single-valued in $D$ and are, moreover, of class $L^{2}(a, D)$. By this procedure, the existence problem can be disposed of by an appeal to a standard compactness argument. The relation between these two types of eigenvalue problems is quite similar to that between the method of the Dirichlet principle [3] and the approach to the fundamental domain functions via the Bergman kernel function $[\mathbf{1}]$. 
THEOREM 1. The eigenvalues $\lambda_{n}$ and eigenfunctions $f_{n}(z)$ satisfy the condition

$$
\begin{aligned}
\iint_{D}\left[\sigma(z) \overline{f_{n}(z)} h(z)\right. & \left.-\lambda_{n}^{-1} \overline{f_{n}^{\prime}(z)} h^{\prime}(z)\right] d x d y \\
& =h(a) \iint_{D} \sigma(z) \overline{f_{n}(z)} d x d y
\end{aligned}
$$

where $h(z)$ is any single-valued regular function in D.

Up to a multiplicative constant our problem is equivalent to that of minimizing the quotient $J_{D}(f) / H_{D}(f)$. Hence with any function $g(z)$ of the class $L^{2}(a, D)$, and any $\epsilon, f(z)=f_{1}(z)+\epsilon g(z)$ is a competing function for which we have the condition

$$
\iint_{D}\left|f_{1}^{\prime}(z)+\epsilon g^{\prime}(z)\right|^{2} d x d y \geq \lambda_{1} \iint_{D} \sigma(z)\left|f_{1}(z)+\epsilon g(z)\right|^{2} d x d y
$$

or, in view of the relation $J_{D}\left(f_{1}\right)=\lambda_{1} H_{D}\left(f_{1}\right)$,

$$
2 \Re\left\{\epsilon \iint_{D}\left(\bar{f}_{1}^{\prime} g^{\rho}-\lambda_{1} \bar{f}_{1} g\right) d x d y\right\} \geq|\epsilon|^{2}[\ldots],
$$

the right side being nonpositive. This inequality evidently holds for every complex value of $\epsilon$ only if

$$
\iint_{D}\left[\overline{f_{1}^{\prime}(z)} g^{\prime}(z)-\lambda_{1} \sigma(z) \overline{f_{1}(z)} g(z)\right] d x d y=0
$$

Similarly obtained are the conditions

$$
\iint_{D}\left[\overline{f_{n}^{\prime}(z)} g^{\prime}(z)-\lambda_{n} \sigma(z) \overline{f_{n}(z)} g(z)\right] d x d y=0 \quad(n=2,3, \cdots),
$$

where $g(z)$ is restricted by the hypothesis

$$
\iint_{D} \sigma(z) g(z) \overline{f_{k}(z)} d x d y=0 \quad(k=1,2, \cdots, n-1) .
$$

It is easily demonstrated that this restriction is unnecessary, however, and consequently (2.7) holds for any function $g(z)$ of $L^{2}(a, D)$. Finally, $(2.6)$ is 
obtained upon setting $g(z)=h(z)-h(a)$.

The fact that the eigenfunctions of $(2.1)$ are a complete set in $L^{2}(a, D)$ follows as in the classical case. Although there are many such complete sets, our eigenfunctions are distinguished by the following property:

THEOREM 2. The eigenfunctions $f_{n}(z)$ constitute a complete orthonormal. set of functions whose first derivatives are also orthogonal over D and complete in the space of functions with single-valued integrals and finite norm. If the eigenvalues are not multiple, this system is unique up to a factor of unit absolute value.

Replacing $g(z)$ with $f_{k}(z)$ in $(2.7)$ yields the identity

$$
\iint_{D} \overline{f_{n}^{\prime}(z)} f_{k}^{\prime}(z) d x d y=\lambda_{n} \iint_{\sigma}(z) \overline{f_{n}(z)} f_{k}(z) d x d y=0,
$$

which, combined with the property of completeness, implies that the first derivatives of the eigenfunctions constitute a complete orthogonal set. Essentially there is but one such system with given normalization whose derivatives are also orthogonal, provided the eigenvalues are not multiple. Indeed, let $\left\{F_{n}(z)\right\}$ be another such system so ordered that $\mu_{1}<\mu_{2}<\cdots$, where

$$
\mu_{n}=\iint_{D}\left|F_{n}^{\prime}(z)\right|^{2} d x d y
$$

and let $\left\{f_{n}(z)\right\}$ be the foregoing eigenfunctions. Expanding $f_{1}(z)$ in a series

$$
f_{1}(z)=\sum_{p=1}^{\infty} a_{p} F_{p}(z)
$$

and noting that $\lambda_{1} \leq \mu_{1}<\mu_{2}<\cdots$, we have

$$
\frac{\iint_{D}\left|f_{1}^{\prime}(z)\right|^{2} d x d y}{\iint_{D} \sigma\left|f_{1}(z)\right|^{2} d x d y}=\frac{\sum_{p=1}^{\infty}\left|a_{p}\right|^{2} \mu_{p}}{\sum_{p=1}^{\infty}\left|a_{p}\right|^{2}} \geq \frac{\mu_{1} \sum_{p=1}^{\infty}\left|a_{p}\right|^{2}}{\sum_{p=1}^{\infty}\left|a_{p}\right|^{2}}=\mu_{1} \geq \lambda_{1},
$$

with equality holding only if $a_{2}=a_{3}=\cdots=0$ and $a_{1}=e^{i \phi}$. Proceeding by induction, we assume that

$$
f_{n-1}(z)=e^{i \theta} F_{n-1}(z)
$$


is orthogonal to $F_{k}(z)(k=1, \cdots, n-1)$; consequently, since

$$
f_{n}(z)=\sum_{m=n}^{\infty} a_{m} F_{m}(z),
$$

we obtain

$$
\frac{\iint_{D}\left|f_{n}^{\prime}(z)\right|^{2} d x d y}{\iint_{D} \sigma\left|f_{n}(z)\right|^{2} d x d y}=\frac{\sum_{m=n}^{\infty}\left|a_{m}\right|^{2} \mu_{m}}{\sum_{m=n}^{\infty}\left|a_{m}\right|^{2}} \geq \mu_{n} \geq \lambda_{n},
$$

with equality holding only if

$$
a_{n+1}=a_{n+2}=\cdots=0 \text { and }\left|a_{n}\right|=1 .
$$

In case multiple eigenvalues exist, however, the preceding proof is invalid and there is no unique system.

3. Examples. Let $G$ be a circle of radius $r$ with center at the origin. If the reference point $a$ is also at the origin and $\sigma \equiv 1$, a closed orthonormal system for $L^{2}(0, G)$ is

$$
\left[\frac{n+1}{\pi}\right]^{1 / 2} \frac{z^{n}}{r^{n+1}}
$$

$$
(n=1,2, \cdots)
$$

The derivatives of this system are also orthogonal; consequently, in virtue of Theorem 2, (3.1) is the set of eigenfunctions, the associated eigenvalues being

$$
\lambda_{n}=\frac{n(n+1)}{r^{2}} .
$$

An example of an orthonormal system in the class $L^{2}(D)$, that is, the functions such that

$$
\iint_{D} f_{n}(z) d x d y=0,
$$

is the set

$$
\begin{aligned}
& f_{n}(z)=z^{n}\left[(n+1) / \pi\left(R^{2 n+2}-r^{2 n+2}\right)\right]^{1 / 2} \quad(n=\cdots,-2,1,2, \cdots), \\
& f_{-1}(z)=z^{-1}[1 / 2 \pi \log (R / r)]^{1 / 2}
\end{aligned}
$$


on the annulus $r<|z|<R$. This system is known to be complete, and the system $\left\{f_{n}^{\prime}(z)\right\}$ is orthogonal; thus, $\left\{f_{n}(z)\right\}$ is the system of eigenfunctions with the corresponding eigenvalues

$$
\begin{aligned}
& \lambda_{n}=n(n+1)\left[\left(R^{2 n}-r^{2 n}\right) /\left(R^{2 n+2}-r^{2 n+2}\right)\right] \quad(n \neq-1,0), \\
& \lambda_{-1}=\left(R^{2}-r^{2}\right) / 2 R^{2} r^{2} \log (R / r) .
\end{aligned}
$$

4. The integral equation. In this section we shall demonstrate that our eigenvalue problem is equivalent to the solution of the homogeneous integral equation

$$
f_{n}(w)=\lambda_{n} \iint_{D} \sigma(z) \overline{K(z, w, a)} f_{n}(z) d x d y,
$$

where the kernel $K(z, w, a)$ is intimately related to the classical domain functions and has been the subject of previous investigations [2;6].

We assume henceforth that $D$ is a finite domain whose boundary $C$ consists of $n$ closed analytic curves $C_{k}(k=1, \ldots, n)$. In the formulas to follow we set $\sigma \equiv 1$ as no generalization accrues otherwise.

The equivalence of the solutions of our eigenvalue problem to those of (4.1) is established by means of the fundamental condition (2.7) and an auxiliary extremal problem. Consider therefore the minimum of the integral

$$
\iint_{D}\left|g^{\prime}(z)\right|^{2} d x d y
$$

for those functions of $L^{2}(a, L)$ for which $g(w)=1$. It is easily established that the minimizing function $K(z, w, a) / K(w, w, a)$ is Hermitian; that is,

$$
\overline{K(z, w, a)}=K(w, z, a)
$$

and (for any function $g(z)$ such that (4.2) is finite) has the reproducing property

$$
g(w)-g(a)=\iint_{D} \overline{K^{\prime}(z, w, a)} g^{\prime}(z) d x d y \text {. }
$$

$K(z, w, a)$ is closely related to the Bergman kernel function $K(z, w)$ with the characteristic reproducing property [1] 


$$
g^{\prime}(w)=\iint_{D} \overline{K(z, w)} g^{\prime}(z) d x d y
$$

Indeed, differentiating (4.3) with respect to $w$, we find

$$
K(z, w)=\frac{\partial^{2} K(z, w, a)}{\partial z \partial \bar{w}}
$$

that is,

$$
K(z, w, a)=\int_{a}^{w} \int_{a}^{z} K(z, w) d z d \bar{w}
$$

It is well known $[6]$ that

$$
2 \pi K(z, w, a)=P(z, w, a)-Q(z, w, a),
$$

where $P(z, w, a)$ and $Q(z, w, a)$ are, respectively, the logarithms of two univalent functions the first of which maps $D$ on the whole complex plane slit along concentric circular arcs around the origin, whereas the second maps $D$ on the full plane furnished with rectilinear slits directed towards the origin. In each case the point $w \in D$ is mapped onto the origin, while the point $a$ of $D$, where the residue of the simple pole is 1 , corresponds to the point at infinity.

The desired integral equation now follows; for, since $K(z, w, a)$ belongs to the class $L^{2}(a, D)$, the reproducing property $(4.3)$ in conjunction with (2.7) yields

$$
\begin{aligned}
f_{n}(w) & =\iint_{D} \overline{K^{\prime}(z, w, a)} f_{n}^{\prime}(z) d x d y \\
& =\lambda_{n} \iint_{D} \overline{K(z, w, a)} f_{n}(z) d x d y .
\end{aligned}
$$

Thus the solutions of the extremal problem are among those of the integral equation (4.5). That the converse is true can be seen as follows. First, the fundamental identity $(2.7)$ can be obtained by forming the scalar product of the derivative of any function $g(z) \in L^{2}(a, D)$ with the derivative

$$
f_{n}^{\prime}(w)=\lambda_{n} \iint_{D} K^{\prime}(w, z, a) f_{n}(z) d x d y
$$


of (4.5). Then in view of (4.3) we obtain

$$
\begin{aligned}
& \iint_{D} \overline{f_{n}^{\prime}(w)} g^{\prime}(w) d u d v \\
& \quad=\lambda_{n} \iint_{D}\left[\iint_{D} \overline{K^{\prime}(w, z, a)} \overline{f_{n}(z)} d x d y\right] g^{\prime}(w) d u d v \\
& \quad=\lambda_{n} \iint_{D} \overline{f_{n}(z)}\left[\iint_{D} \overline{K^{\prime}(w, z, a)} g^{\prime}(w) d u d v\right] d x d y \\
& \quad=\lambda_{n} \iint_{D} \overline{f_{n}(z)} g(z) d x d y .
\end{aligned}
$$

Now, if $f_{n}(z)$ and $f_{m}(z)$ are two solutions of the integral equation (4.1), and $\lambda_{n}$ and $\lambda_{m}$ are the corresponding eigenvalues, it follows from the Hermitian character of $K(z, w, a)$ that

$$
\left(\lambda_{n}-\overline{\lambda_{m}}\right) \iint_{D} f_{n}(z) \overline{f_{m}(z)} d x d y=0
$$

Setting $g(z)=f_{n}(z)$ in (4.7), we obtain

$$
\iint_{D}\left|f_{n}^{\prime}(z)\right|^{2} d x d y=\lambda_{n} \iint_{D}\left|f_{n}(z)\right|^{2} d x d y
$$

which shows that all eigenvalues are positive. Hence (4.8) yields

$$
\iint_{D} f_{n}(z) \overline{f_{m}(z)} d x d y=0
$$

if $\lambda_{n} \neq \lambda_{m}$. In view of (4.7) this entails

$$
\iint_{D} f_{n}^{\prime}(z) \overline{f_{m}^{\prime}(z)} d x d y=0
$$

But, by Theorem 2, (4.9) and (4.10) characterize, up to multiplicative constants, the solutions of the eigenvalue problem treated in $\$ 2$. Summing up, we have:

THEORЕм 3. The eigenvalues and eigenfunctions of the extremal problem 


$$
\frac{\iint_{D}\left|f^{\prime}(z)\right|^{2} d x d y}{\iint_{D}|f(z)|^{2} d x d y}=\lambda=\text { minimum, } \quad f(a)=0
$$

are identical with those of the integral equation

$$
f(w)=\lambda \iint_{D} \overline{K(z, w, a)} f(z) d x d y,
$$

where the Hermitian kernel $K(z, w, a)$ is constructed from the Bergman kernel function by means of (4.4).

It is worth noting that

$$
\iint_{D} \iint_{D}|K(z, w, a)|^{2} d x d y d u d v<\infty
$$

and therefore that $K(z, w, a)$, unlike the Bergman kernel $K(z, w)$, is a regular kernel in the sense of the Hilbert theory of integral equations. We also point out that if $D$ is bounded by closed analytic curves, the eigenfunctions $f_{n}(z)$ are regular in the closure of $D$. In this case $\overline{K(z, w, a)}$ is, as a function of $w$, regular in a domain $D^{\prime}$ which contains $D$ and whose boundary has no points in common with that of $D$. The right side of (4.11) represents the function $f(w)$ for $w \in D$ and another analytic function-say $f_{1}(w)$ - for $w \in D^{\prime}-D$. If $w$ crosses the boundary $C$ of $D$ and $z \in D, \overline{K(z, w, a)}$ remains regular; if $z$ is a point of $C$, the singular behavior of $\overline{K(z, w, a)}$ on crossing $C$ is essentially that of $\log \left(w^{\prime}-\bar{z}\right)$, where $w^{\prime} \notin D$ if $w \in D$, and $w^{\prime}$ and $w$ become symmetric points if a suitable section of $C$ is conformally mapped onto a linear segment [cf. 6]. From these properties of $\overline{K(z, w, a)}$ it is easily inferred that the right side of (4.11) is a continuous function of $w$ if $w$ crosses $C$ and if $w$ varies along $C$. Hence $f(w)$ and $f_{1}(w)$ are analytic continuations of the same analytic function, and $f(w)$ is thus regular on $C$.

5. Examples. The Bergman kernel function for the annulus $0<r<|z|<1$ is

$$
K(z, w)=\frac{1}{\pi} \sum_{m=-\infty}^{\infty} \frac{m(z \bar{w})^{m-1}}{1-r^{2 m}},
$$

the prime indicating that $m \neq 0$ in the summation. The integral equation characterizing the solutions of our problem is then 


$$
f_{n}(w)=\frac{\lambda_{n}}{\pi} \iint_{D} \sum_{m=-\infty}^{\infty} \frac{\overline{\left(z^{m}-a^{m}\right)}\left(w^{m}-a^{m}\right)}{m\left(1-r^{2 m}\right)} f_{n}(z) d x d y .
$$

With the substitutions

$$
\omega_{1}=i \pi, \omega_{2}=\log r \text {, and } q=\exp \left(i \omega_{2} / \omega_{1}\right)=r \text {, }
$$

the kernel in (5.1) can be expressed in closed form with the help of the Weierstrass $\zeta$-function; namely,

$$
K(z, w, a)=\frac{1}{\pi}[\zeta(\log a \bar{w})-\zeta(\log z \bar{w})+\zeta(\log z \bar{a})-\zeta(\log a \bar{a})] .
$$

For the circle $|z|<r$ the Bergman kernel is

$$
K(z, w)=\frac{r^{2}}{\pi\left(r^{2}-z \bar{w}\right)^{2}} ;
$$

consequently, by (4.4) we obtain

$$
K(z, w, a)=\frac{1}{\pi} \sum_{n=1}^{\infty} \frac{\left[(z / r)^{n}-(a / r)^{n}\right]\left[\overline{(w / r)^{n}-(a / r)^{n}}\right]}{n}
$$

or, in closed form,

$$
K(z, w, a)=\frac{1}{\pi} \log \frac{\left(r^{2}-z \bar{a}\right)\left(r^{2}-a \bar{w}\right)}{\left(r^{2}-\bar{w} z\right)\left(r^{2}-a \bar{a}\right)} .
$$

If the origin of the circle is taken as the reference point, it is easily verified that the characteristic functions and constants of the equation

$$
f_{n}(w)=\frac{-\lambda_{n}}{\pi} \iint_{|z|<1} \overline{\log (1-\bar{w} z)} f_{n}(z) d x d y
$$

are, respectively,

$$
f_{n}(z)=z^{n}[(n+1) / \pi]^{1 / 2}
$$$$
(n=1,2, \ldots)
$$

and 


$$
\lambda_{n}=n(n+1)
$$

From the known transformation law for the Bergman kernel [1] it is easily ascertained that $K(z, w, a)$ is a conformal invariant; consequently, the integral equation for the characteristic functions and constants of any simply connected domain can be derived from (5.2).

6. Variation of the domain functions. Let $D^{*}$, a domain with the same degree of connectivity as $D$, be obtained from $D$ by shifting each boundary point $z(s)$ along the exterior normal by an anount $\delta n=\eta v(s)$, where $v(s)$ is a continuous function on $C$ and $\eta$ is sufficiently small.

The corresponding first-order variation of the Bergman kernel function was found by Schiffer $[\mathbf{1 0}]$ to be

$$
\delta K(z, w)=-\int_{C} \overline{K(t, z)} K(t, w) \delta n d s .
$$

Combining (6.1) with (4.4) we obtain the formula

$$
\delta K(z, w, a)=-\int_{C} K^{\prime}(t, w, a) \overline{K^{\prime}(t, z, a)} \delta n d s
$$

expressing the variation of the kernel of (4.11).

Using this variational formula we may obtain corresponding variation formulas for the eigenfunctions and eigenvalues of (4.11). Since we are not going to employ these formulas in the sequel, we shall content ourselves with a formal derivation of the variation formula for the eigenvalues, omitting the rather lengthy discussion required to make the proof entirely rigorous.

We first note that, since $f_{n}(z)$ is regular on $C$, it follows from (6.2) that

$$
\begin{aligned}
& \iint_{D} \iint_{D} \delta K(z, w, a) f_{q}(w) \overline{f_{m}(z)} d x d y d u d v \\
& \quad=-\int_{C}\left[\iint_{D} K^{\prime}(t, w, a) f_{q}(w) d u d v\right]\left[\iint_{D} \overline{K^{\prime}(t, z, a) f_{m}(z)} d x d y\right] \delta n d s \\
& =\frac{-1}{\lambda_{q} \lambda_{m}} \int_{C} f_{q}^{\prime}(t) \overline{f_{m}^{\prime}(t)} \delta n d s
\end{aligned}
$$

We next assume that $D^{*} \supset D$. If the quantities belonging to $D^{*}$ are denoted by asterisks, we have then, in view of (4.11), 


$$
\begin{aligned}
f_{n}^{*}(w)-f_{n}(w) & =\iint_{D}\left[\lambda_{n}^{*} \overline{K^{*}(z, w, a)} f_{n}^{*}(z)-\lambda_{n} \overline{K(z, w, a)} f_{n}(z)\right] d x d y \\
& +\lambda_{n}^{*} \iint_{D^{*}-D} \overline{K^{*}(z, w, a)} f_{n}^{*}(z) d x d y
\end{aligned}
$$

$\Lambda$ ssuming, without proof, that $f_{n}^{*}(z)^{*}$ and $\lambda_{n}^{*}$ differ from $f_{n}(z)$ and $\lambda_{n}$, respectively, only by quantities of order $\eta$, we find the last integral to be of the form

$$
\lambda_{n} \int_{C} \overline{K(t, w, a)} f_{n}(t) \delta n d s+o(\eta)
$$

whence (6.4) leads to

$$
\begin{aligned}
\delta f_{n}(w) & =\lambda_{n} \iint_{D} \overline{\delta K(z, w, a)} f_{n}(z) d x d y+\lambda_{n} \iint_{D} \overline{K(z, w, a)} \delta f_{n}(z) d x d y \\
& +\delta \lambda_{n} \iint_{D} \overline{K(z, w, a)} f_{n}(z) d x d y+\lambda_{n} \int_{C} \overline{K(t, w, a)} f_{n}(t) \delta n d s,
\end{aligned}
$$

where terms of order $o(\eta)$ have been neglected. We now multiply this identity by $\overline{f_{n}(w)}$ and integrate over $D$. Simplifying the result by means of $(4.10)$ and (6.3), we obtain

$$
\begin{aligned}
\iint_{D} \overline{f_{n}(w)} \delta f_{n}(w) d u d v & =\frac{-1}{\lambda_{n}} \int_{C}\left|f_{n}^{\prime}(t)\right|^{2} \delta n d s+\iint_{D} \overline{f_{n}(z)} \delta f_{n}(z) d x d y \\
& +\frac{\delta\left(\lambda_{n}\right)}{\lambda_{n}} \iint_{D}\left|f_{n}(w)\right|^{2} d u d v+\int_{C}\left|f_{n}(t)\right|^{2} \delta n d s .
\end{aligned}
$$

Hence, if $f_{n}(z)$ is normalized by the condition

$$
\iint_{D}\left|f_{n}(z)\right|^{2} d x d y
$$

we arrive at the following result:

THEOREM 4. If the domain $D$ is made subject to a first-order normal variation $\delta n$, the corresponding variation of the eigenvalues $\lambda_{m}$ is given by

$$
\delta\left(\lambda_{m}\right)=\int_{C}\left[\left|f_{m}^{\prime}(t)\right|^{2}-\lambda_{m}\left|f_{m}(t)\right|^{2}\right] \delta n d s,
$$


where $f_{m}(z)$ is the eigenfunction associated with $\lambda_{m}$.

The extension of this result from the case $D^{*} \supset D$ to the general case is easily carried out by means of the artifice employed by Hadamard for a similar purpose in the derivation of his variation formula for the Green's function [8].

Formula (6.5) shows that no general monotonicity property of the eigenvalues can be expected. For with the notation

$$
A(t) \equiv\left|f_{m}^{\prime}(t)\right|^{2}-\lambda_{m}\left|f_{m}(t)\right|^{2},
$$

ive have

$$
\iint_{D} A(z) d x d y=0
$$

which shows that $A(t)$ takes both positive and negative values in $D$. If we write $(6.5)$ in the form

$$
\delta\left(\lambda_{m}\right)=\int_{C} A(t) \delta n d s,
$$

it is thus clear that the assumption $\delta n \geq 0$ ( or $\delta n \leq 0$ ) for $t \in C$ cannot guarantee a definite sign for the corresponding variation of $\lambda_{m}$.

7. The resolvent kernel. Consider now the inhomogeneous equation

$$
f(w)=h(w)+\lambda \iint_{D} \overline{K(z, w, a)} f(z) d x d y
$$

and its associated resolvent kernel

$$
K(z, w ; \lambda)=\sum_{n=1}^{\infty} \frac{f_{n}(z) \overline{f_{n}(w)}}{\lambda_{n}-\lambda} .
$$

Wie shall show first that $K(z, w ; \lambda)$ can be characterized by means of a minimum problem and constructed directly from a complete system of complex orthogonal functions. Thus, consider the following extremal problem: in the class $L^{2}(a, D)$ determine the function $g(z)$, regular in $D$ with $g(w)=1$ at a fixed point $w$ of $D$, which minimizes the integral

$$
J_{\lambda}(g)=\iint_{D}\left[\left|g^{\prime}(z)\right|^{2}-\lambda|g(z)|^{2}\right] d x d y,
$$


$\lambda$ being less than the smallest eigenvalue of the extremal problem (1.1). For this class of functions we have

$$
\frac{\iint_{D}\left|g^{\prime}(z)\right|^{2} d x d y}{\iint_{D}|g(z)|^{2} d x d y} \geq \lambda_{1}>\lambda ;
$$

hence,

$$
\text { g.l.b. } J_{\lambda}(g)=A>0 \text {. }
$$

Finally, this class of functions is compact, and therefore a function $g(z)$ exists such that $J_{\lambda}(g)=A$.

Now orthonormalize a closed system of functions $\left\{p_{n}(z)\right\}$ so that the conditions

$$
\iint_{D}\left[p_{n}^{\prime}(z) \overline{p_{m}^{\prime}(z)}-\lambda p_{n}(z) \overline{p_{m}(z)}\right] d x d y=\delta_{n m}
$$

are satisfied. The associated kernel

$$
K^{\prime}=\sum_{n=1}^{\infty} p_{n}(z) \overline{p_{n}(w)}
$$

converges uniformly and absolutely in every closed subdomain of $D$ and provides the minimum of (7.3) when normalized. Moreover, it is easily verified that $K$ reproduces according to the formula

$$
h(w)=\iint_{D}\left[\overline{K^{\prime}} h^{\prime}(z)-\lambda \bar{K} h(z)\right] d x d y .
$$

The uniqueness of a kernel $K$ with the reproducing property (7.4) follows from the definiteness of the expression (7.3) by a standard argument. Also it is easy to see that $K$ is identical with the resolvent kernel $K(z, w ; \lambda)$ and that, consequently, the solution of the inhomogeneous equation (7.1) is

$$
f(w)=\iint_{D} \overline{K^{\prime}(z, w ; \lambda)} h^{\prime}(z) d x d y .
$$

Indeed, the functions 


$$
p_{n}(z)=\left[\lambda_{n}-\lambda\right]^{-1 / 2} f_{n}(z) \quad(n=1,2, \ldots),
$$

where $f_{n}(z)$ and $\lambda_{n}$ are the $n$th eigenfunction and eigenvalue of our original extremal problem, are normalized in the sense of the above metric; that is,

$$
\begin{aligned}
& \iint_{D}\left[p_{n}^{\prime}(z) \overline{p_{m}^{\prime}(z)}-\lambda p_{n}(z) \overline{p_{m}(z)}\right] d x d y \\
& \quad=\left[\left(\lambda_{n}-\lambda\right)\left(\lambda_{m}-\lambda\right)\right]^{-1 / 2} \iint_{D}\left(f_{n}^{\overline{f_{i n}^{\prime}}}-\lambda f_{n} \overline{f_{m}}\right) d x d y=\delta_{n m} .
\end{aligned}
$$

Therefore, the kernel is

$$
K=K(z, w ; \lambda)=\sum_{n=1}^{\infty} p_{n}(z) \overline{p_{n}(w)}=\sum_{n=1}^{\infty} \frac{f_{n}(z) \overline{f_{n}(w)}}{\lambda_{n}-\lambda}
$$

which is identical with (7.2).

In view of their reproducing properties, as well as in other aspects, it appears that the resolvent kernel is closely allied to the eigenfunctions of (1.1). More precisely, we have:

THEOREM 5. Let $\left\{f_{n}(z)\right\}$ and $\lambda_{n}$ be the system of eigenfunctions and eigenvalues associated with the class $L^{2}(D)$; that is,

$$
\iint_{D} f_{n}(z) d x d y=0
$$

If $F_{n}(z)$ and $\mu_{n}$, respectively, represent the nth eigenfunction and eigenvalue associated with a point $w \in D$ and such that $\iint_{D} F_{n}(z) d x d y \neq 0$, then

$$
F_{n}(z) / \iint_{D} F_{n}(z) d x d y=c-\mu_{n} K\left(z, w ; \mu_{n}\right),
$$

where

$$
c^{-1}=\iint_{D} d x d y
$$

$K\left(z, w ; \mu_{n}\right)$ is the resolvent kernel 


$$
K\left(z, w ; \mu_{n}\right)=\sum_{p=1}^{\infty} \frac{f_{p}(z) \overline{f_{p}(w)}}{\lambda_{p}-\mu_{n}},
$$

and $\mu_{n}$ is determined as the nth root (in order of magnitude) of

$$
\mu_{n} K\left(w, w ; \mu_{n}\right)=c
$$

Proof. Let $g(z)$ be any regular function and set

$$
h(z)=g(z)-M c,
$$

where

$$
M=\iint_{D} g(z) d x d y \text {. }
$$

Accordingly, $h(z)$ is a function of the class $L^{2}(D)$ reproduced by the resolvent kernel $K(z, w ; \mu), \mu \neq \lambda_{n}$; namely,

$$
h(w)=\iint_{D}\left[\overline{K^{\prime}} h^{\prime}(z)-\mu \bar{K} h(z)\right] d x d y .
$$

Now with

$$
H_{n}(z)=c-\mu_{n} K\left(z, w ; \mu_{n}\right)
$$

we obtain the identity

$$
\begin{aligned}
& \iint_{D}\left[\overline{H_{n}(z)} g(z)-\mu_{n}^{-1} \overline{H_{n}^{\prime}(z)} g^{\prime}(z)\right] d x d y \\
& \quad=\iint_{D}\left\{\left[c-\mu_{n} \bar{K}\right][h(z)+M c]-\mu_{n}^{-1}\left[-\mu_{n} \overline{K^{\prime}}\right] h^{\prime}(z)\right\} d x d y \\
& =M c^{2} \iint_{D} d x d y-\mu_{n} M c \iint_{D} \bar{K} d x d y+c \iint_{D} h(z) d x d y \\
& \quad+\iint_{D}\left[\overline{K^{\prime}} h^{\prime}(z)-\mu_{n} \bar{K} h(z)\right] d x d y \\
& =M c+h(w)=g(w) .
\end{aligned}
$$


Setting, in particular, $g(z)=F_{m}(z)$, we obtain

$$
\iint_{D}\left(\overline{H_{n}} F_{m}-\mu_{n}^{-1} \overline{H_{n}^{\prime}} F_{m}^{\prime}\right) d x d y=F_{m}(w)=0 \text {. }
$$

On the other hand, it follows from $(2.6),(7.5)$, and ( 7.6$)$ that

$$
\iint_{D}\left(\overline{H_{n}} F_{m}-\mu_{m}^{-1} \overline{H_{n}^{\prime}} F_{m}^{\prime}\right) d x d y=\overline{H_{n}(w)} \iint_{D} F_{m} d x d y=0
$$

Combining this with (7.8), we obtain

$$
\left(\frac{1}{\mu_{n}}-\frac{1}{\mu_{m}}\right) \iint_{D} \overline{H_{n}^{\prime}} F_{m}^{\prime} d x d y=0
$$

that is, either $\mu_{n}$ coincides with one of the eigenvalues $\mu_{1}, \mu_{2}, \cdots$, or else

$$
\iint_{D} \bar{H}_{n}^{\prime} F_{m}^{\prime} d x d y=0 \quad(m=1,2, \ldots) .
$$

In view of $(7.8)$, the latter alternative would mean

$$
\iint_{D} \bar{H}_{n} F_{m} d x d y=0 \quad(m=1,2, \cdots)
$$

but this is impossible since the functions $F_{1}(z), F_{2}(z), \ldots$ form a complete orthonormal set in $L^{2}(w, D)$, and $H_{n}(z) \not \equiv 0$. This proves that all solutions of (7.5) coincide with eigenvalues $\mu_{m}$.

To prove that, conversely, all eigenvalues are solutions of (7.5), we remark that the reproducing property (7.7) remains valid even though $\mu$ is not a solution of $(7.5)$. If we write

$$
H(z)=c-\mu K(z, w ; \mu)
$$

we have $H(w) \neq 0$, and the right side of (7.9) will not vanish since we have assumed

$$
\iint_{D} F_{m}(z) d x d y \neq 0
$$

Formula (7.10) will therefore be replaced by 


$$
\left(\frac{1}{\mu}-\frac{1}{\mu_{m}}\right) \iint_{D} \overline{H^{\prime}} F_{m}^{\prime} d x d y \neq 0,
$$

which shows that $\mu$ cannot coincide with any value $\mu_{m}$. We have thus proved that, except for the case in which

$$
\iint_{D} F_{m} d x d y=0
$$

the set of eigenvalues coincides with the set of solutions of (7.5).

That the functions $H_{n}(z)$ are, except for constant factors, identical with the eigenfunctions $F_{n}(z)$ follows now from the completeness of the eigenfunctions and the observation that, by (7.8) and (7.10), $H_{m}(z)$ is orthogonal to all $F_{n}(z), n \neq m$.

The spectrum of eigenvalues consists of the roots of the transcendental equation

$$
G(\mu)=c-\mu \sum_{p=1}^{\infty} \frac{\left|f_{p}(w)\right|^{2}}{\lambda_{p}-\mu}=0 .
$$

For $0 \leq \mu \leq \lambda_{1}$, the function $G(\mu)$ varies from $c$ to $-\infty$; similarly in any interval $\lambda_{n-1} \leq \mu \leq \lambda_{n}, G(\mu)$ varies monotonically from $+\infty$ to $-\infty$. Thus, if the problem does not entail multiple eigenvalues, we have proved:

THEOREM 6. Let $\left\{\lambda_{n}\right\}$ be the set of eigenvalues of (1.1) for the class $L^{2}(D)$, while $\left\{\lambda_{n}(w)\right\}$ are those associated with the class $L^{2}(w, D)$. Then setting $\lambda_{0}=0$, we have

$$
\lambda_{n-1} \leq \lambda_{n}(w) \leq \lambda_{n}
$$

for all $w \in D$ and for every $n, n \geq 1$.

8. A boundary relation. The Bergman kernel function and several of its analogues give rise to certain boundary relations existing between pairs of analytic functions [7]. Likewise somewhat similar developments are possible in the case of the kernels which appeared in the preceding sections.

Our point of departure in the derivation of these boundary relations is the reproducing properties (2.6) and (7.4). However, since the procedure involves integration, and since, in the case of multiply connected domains, the eigenfunctions of (1.1) do not in general lie in the class of derivatives of single- 
valued functions, we reformulate our extremal problem for functions of this class, taking $\sigma \equiv 1$. Thus let $f_{n}^{\prime}(z)$ and $\lambda_{n}$ denote the $n$th eigenfunction and the min max, respectively, of the quotient

$$
\frac{\iint_{D}\left|f^{\prime \prime}(z)\right|^{2} d x d y}{\iint_{D}\left|f^{\prime}(z)\right|^{2} d x d y}
$$

with the conditions

$$
\iint_{D} f^{\prime}(z) \overline{v_{j}^{\prime}(z)} d x d y=0 \quad(j=1,2, \cdots, n-1),
$$

and either

$$
f^{\prime}(a)=0,
$$

or

$$
\iint_{D} f^{\prime}(z) d x d y=0
$$

If $D$ is simply connected, (8.1) is, of course, identical with (1.1). Using the abbreviation

$$
A_{n}=\iint_{D} f_{n}^{\prime}(z) d x d y
$$

we see that the immediate analogue of $(2.6)$ is

$$
\overline{A_{n}} h^{\prime}(a)=\iint_{D}\left[\overline{f_{n}^{\prime}} h^{\prime}-\lambda_{n}^{-1} \overline{f_{n}^{\prime \prime}} h^{\prime \prime}\right] d x d y \text {. }
$$

Now it was shown above that $f_{n}^{\prime}(z)$ is regular in the closure of $D$. Hence, if we restrict our attention to functions $h(z)$ such that $h^{\prime \prime}(z)$ is continuous in $D+C$, we may apply Green's formula to (8.2). This yields

$$
\begin{aligned}
\overline{A_{n}} h^{\prime}(a) & =\frac{1}{2 i} \int_{C}\left(\overline{f_{n}} h^{\prime}-\lambda_{n}^{-1} \overline{f_{n}^{\prime}} h^{\prime \prime}\right) d z & \\
& =\frac{1}{2 i} \int_{C} h^{\prime}\left[\overline{f_{n}+\lambda_{n}^{-1} z^{\prime 2} f_{n}^{\prime \prime}}\right] d z, & z^{\prime}=d z /|d z| .
\end{aligned}
$$


It follows that

$$
\int_{C} h^{\prime}(z) B(s) d z=0,
$$

where

$$
B(s)=\pi\left[\overline{f_{n}+\lambda_{n}^{-1} z^{\prime 2} f_{n}^{\prime \prime}}\right]-\frac{\overline{A_{n}}}{z-a},
$$

and $s$ is the length parameter on $C$.

Integrating ( 8.4$)$ by parts, we obtain

$$
\int_{C} h(z) \frac{\partial B}{\partial s} d s=0,
$$

or

$$
\int_{C} h(z)\left[\overline{z^{\prime}} \partial B / \partial s\right] d z=0
$$

This identity holds for any single-valued and regular function $h(z)$ in $D$ for which the integral exists; it is evident that the restriction that $h^{\prime \prime}(z)$ be continuous in $D+C$ can now easily be removed by means of an approximation argument.

It follows [7] from (8.6) that $\bar{z}^{\prime} \partial B / \partial s$ coincides with the boundary values of an analytic function $G(z)$ which is regular and single-valued in $D$; that is,

$$
\bar{z}^{\prime} \partial B / \partial s=G(z)
$$$$
z \in C \text {. }
$$

Hence, if $z_{1}$ and $z_{2}$ are two points on the same boundary components $C_{k}$ of $C$, and $s_{1}, s_{2}$ are the corresponding values of $s$, then

$$
B\left(s_{2}\right)-B\left(s_{1}\right)=\int_{z_{1}}^{z_{2}} G(z) d z .
$$

Since $B$ is single-valued on $C_{k}$, we have

$$
\int_{C_{k}} G(z) d z=0
$$

for every $C_{k}$, which shows that $G(z)$ is the derivative of a single-valued 
function, say $g(z)$. Thus

$$
B\left(s_{2}\right)-B\left(s_{1}\right)=g\left(z_{2}\right)-g\left(z_{1}\right),
$$$$
z_{1}, z_{2} \in C_{k}
$$

or, what amounts to the same thing,

$$
B(s)=g(z)+c_{m},
$$

where $c_{m}$ is a constant. Using the definition (8.5) of $B(s)$, we arrive at the following result.

THEOREM 7. Let the boundary $C$ of $D$ consist of $m$ analytic components $C_{m}$. For $f_{n}^{\prime \prime}(z)$ and $\lambda_{n}$, the nth eigenfunction and eigenvalue, respectively, of (8.1), the boundary relation

$$
\pi\left[\overline{f_{n}(z)+\lambda_{n}^{-1} z^{\prime 2} f_{n}^{\prime \prime}(z)}\right]=\overline{A_{n}} /(z-a)+g(z)+c_{m}
$$

holds, where $c_{m}$ is constant on $C_{m}, g(z)$ is regular in $D$, and

$$
A_{n}=\iint_{D} f_{n}^{\prime}(z) d x d y
$$

Differentiating the boundary relation (8.3) with respect to $s$, we obtain

$$
\begin{array}{r}
\overline{\left[f_{n}^{\prime}(z)+\lambda_{n}^{-1} 2 z^{\prime \prime} f_{n}^{\prime \prime}(z)+\lambda_{n}^{-1} z^{\prime 2} f_{n}^{\prime \prime \prime}(z)\right] d z} \\
=\left[\frac{-\overline{A_{n}}}{\pi(z-a)^{2}}+g(z)\right] d z .
\end{array}
$$

This identity can be used, in some cases, for the effective computation of the functions $f_{n}(z)$. It is well known that the Bergman kernel function $K(z, a)$ of $D$ can be uniquely characterized by the existence of a boundary relation of the form

$$
\overline{K(z, a) d z}=\left[\frac{-1}{\pi(z-a)^{2}}+p^{\prime}(z)\right] d z
$$

where $p(z)$ is regular and single-valued in $D[1]$. Comparing this with (8.4), we find that in those cases in which both $z^{\prime 2}$ and $z^{\prime \prime}$ coincide with the boundary values of regular and single-valued analytic functions, the function 


$$
w_{n}(z)=f_{n}^{\prime}(z) / A_{n}
$$

will be a solution of the differential equation

$$
z^{\prime 2} w_{n}^{\prime \prime}(z)+2 z^{\prime \prime} w_{n}^{\prime}(z)+\lambda_{n} w_{n}(z)=\lambda_{n} K(z, a),
$$

if $z^{\prime 2}$ and $z^{\prime \prime}$ are replaced by the analytic functions in question.

If $D$ is the unit circle, we have

$$
z^{\prime 2}=-z^{2} \text { and } z^{\prime \prime}=-z
$$

The Bergman kernel is in this case of the form

$$
K(z, a)=\pi(1-\bar{a} z)^{-2}
$$

Hence ( 8.5 ) yields the differential equation

$$
w_{n}^{\prime \prime}+\frac{2}{z} w_{n}^{\prime}-\frac{\lambda_{n}}{z^{2}} w_{n}=\frac{-\lambda_{n}}{\pi z^{2}(1-\bar{a} z)^{2}} .
$$

The solution of the corresponding homogeneous equation is $c_{1} z^{\alpha}+c_{2} z^{\beta}$, where

$$
\alpha=-\frac{1}{2}+\sqrt{\frac{1}{4}+\lambda_{n}}, \quad \beta=-\frac{1}{2}-\sqrt{\frac{1}{4}+\lambda_{n}} .
$$

If $\alpha, \beta$ are not integers, a solution of $(8.6)$ is

$$
w_{n}(z)=\frac{-\lambda_{n}}{\pi} \sum_{p=0}^{\infty} \frac{(p+1)(z \bar{a})^{p}}{\left[p(p+1)-\lambda_{n}\right]} .
$$

If $\alpha, \beta$ are integers - that is, in view of (8.7), if $\lambda_{n}=n(n+1)$, where $n$ is an integer - this solution will contain a logarithmic term unless $a=0$. Since $w_{n}(z)$ must be regular in $|z|<1$, we must have for $a \neq 0$,

$$
\lambda_{n} \neq n(n+1)
$$

In this case both $z^{\alpha}$ and $z^{\beta}$ are not regular at $z=0$, and the eigenfunctions are therefore given by (8.8), while the eigenvalues are determined by the condition $w_{n}(a)=0$; that is, 


$$
\sum_{p=0}^{\infty} \frac{(p+1)|a|^{2 p}}{\left[p(p+1)-\lambda_{n}\right]}=0 .
$$

If $a=0$, the general solution of (8.6) is $c_{1} z^{\alpha}+c_{2} z^{\beta}+\pi^{-1}$, and we shall thus have a regular solution only if $\lambda_{n}=n(n+1)$ and $c_{2}=0(\alpha>\beta)$. Thus, the eigenvalues are in this case the numbers $n(n+1)$ in agreement with the results of $\leqslant 3$.

Similarly, if $K(z, a)$ is the Bergman kernel function of any domain $D$ bounded by slits which are parts of the same circle about the origin, the differential equation, like (8.6), is

$$
w^{\prime \prime}(z)+\frac{2}{z} w^{\prime}(z)-\frac{\lambda}{z^{2}} w(z)=\frac{-\lambda}{z^{2}} K(z, a) .
$$

On the annulus $r<|z|<R$ the solution for an arbitrary point $a$ of $D$ is most easily obtained by a direct application of Theorem 5 . The eigenvalues and eigenfunctions, respectively, associated with the space $L^{2}(D)$ are

$$
\begin{aligned}
f_{n}(z) & =z^{n}\left[\frac{(n+1)}{\pi\left(R^{2 n+2}-r^{2 n+2}\right)}\right]^{1 / 2} \quad(n \neq 0,1), \\
f_{-1}(z) & =\frac{1}{z[2 \pi \log (R / r)]^{1 / 2}},
\end{aligned}
$$

and

$$
\begin{aligned}
\lambda_{n} & =\frac{n(n+1)\left(R^{2 n}-r^{2 n}\right)}{R^{2 n+2}-r^{2 n+2}}, \\
\lambda_{-1} & =\frac{R^{2}-r^{2}}{2 R^{2} r^{2} \log (R / r)} .
\end{aligned}
$$

Hence the resolvent kernel is

$$
\begin{aligned}
K(z, w ; \mu) & =R^{2} r^{2} / \pi z \bar{w}\left[R^{2}-r^{2} 2 \mu R^{2} r^{2} \log (R / r)\right] \\
& +\frac{1}{\pi} \sum_{p=-\infty}^{\infty} \frac{(p+1)(z \bar{w})^{p}}{R^{2 p}\left(p^{2}+p-\mu R^{2}\right)-r^{2 p}\left(p^{2}+p-\mu r^{2}\right)} \quad(p \neq-1,0) .
\end{aligned}
$$


Thus, for the $n$th eigenfunction $F_{n}(z)$ we have

$$
\begin{aligned}
F_{n}(z) / \iint_{D} F_{n}(z) d x d y=c-\mu_{n} K\left(z, w ; \mu_{n}\right) \\
=-\mu_{n} R^{2} r^{2} / \pi z \bar{w}\left[R^{2}-r^{2}-2 \mu_{n} R^{2} r^{2} \log (R / r)\right] \\
\quad-\frac{\mu_{n}}{\pi} \sum_{p=-\infty}^{\infty} \frac{(p+1)(z \bar{w})^{p}}{R^{2 p}\left(p^{2}+p-\mu_{n} R^{2}\right)-r^{2 p}\left(p^{2}+p-\mu_{n} r^{2}\right)}(p \neq-1),
\end{aligned}
$$

with the condition $F_{n}(a)=0$ determining the eigenvalues.

Another case to which (8.5) can be applied is that of a domain bounded by parallel rectilinear slits. Taking, for simplicity, these slits to be horizontal, we have

$$
z^{\prime 2}=1 \text { and } z^{\prime \prime}=0
$$

both analytic functions of $z$ regular in $D$. Hence (8.5) takes the form

$$
w^{\prime \prime}(z)+\lambda w(z)=\lambda K(z, a) \text {. }
$$

For the half plane $d\{z\}>0$, the Bergman kernel is $-1 / \pi(z-\bar{a})^{2}$; consequently, the eigenfunctions are of the form

$$
\begin{aligned}
w(z)= & \pi^{-1} \sqrt{\lambda} \cos \sqrt{\lambda} z \int_{a}^{z}(z-\bar{a})^{-2} \sin \sqrt{\lambda} z d z \\
& -\pi^{-1} \sqrt{\lambda} \sin \sqrt{\lambda} z \int_{a}^{z}(z-\bar{a})^{-2} \cos \sqrt{\lambda} z d z
\end{aligned}
$$

\section{REFERENCES}

1. Stephen Bergman, The kernel function and conformal mapping, American Mathematical Society, New York, 1950.

2. Stephen Bergman, and M. Schiffer, Kernel functions and conformal mapping, Compositio Math. 8 (1951), $205-249$.

3. R. Courant, Dirichlet's principle, conformal mapping and minimal surfaces, Interscience Publishers, Inc., New York, 1950.

4. R. Courant, and D. Hilbert, Methoden der Mathematischen Physik I, Julius Springer, Berlin, 1937.

5. P. R. Garabedian, A partial differential equation arising in conformal mapping, Pacific J. Math. 1 (1951), 485-524. 
6. P.R. Garabedian and M. Schiffer, Identities in the theory of conformal mapping, Trans. Amer. Math. Soc. 65 (1949), $187-238$.

7. - On existence theorems of potential theory and conformal mapping, Ann. of Math. 52 (1950), 164-187.

8. J. Hadamard, Sur les operations fonctionnelles, C. R. Acad. Sci. Paris, 136 (1903), $351-354$.

9. O. Lehto, Anwendung orthogonaler Systeme auf gewisse funktionen theoretische Extremal-und Abbildungs probleme, Ann. Acad. Sci. Fennicae. Ser. A, No. 59, 1949.

10. M. Schiffer, The kernel function of an orthonormal system, Duke Math. J., 13 (1946), 529-540.

Lousiana State University 



\section{PACIFIC JOURNAL OF MATHEMATICS}

\section{EDITORS}

\author{
M.M. SCHIFFE R* \\ Stanford University \\ Stanford, California \\ E. HEWITT \\ University of Washington \\ Seattle 5, Washington
}

\author{
R.P. DILWORTH \\ Calif ornia Institute of Technology \\ Pasadena 4, California \\ E. F. BECKENBACH** \\ University of California \\ Los Angeles 24, California
}

\section{ASSOCIATE EDITORS}

$\begin{array}{llll}\text { H. BUSEMANN } & \text { P.R. HALMOS } & \text { BØRGE JESSEN } & \text { J. J. STOKER } \\ \text { HERBERT FEDERER } & \text { HEINZ HOPF } & \text { PAUL LÉVY } & \text { E. G. STRAUS } \\ \text { MARSHALL HALL } & \text { R.D. JAMES } & \text { GEORGE PÓLYA } & \text { KÔSAKU YOSIDA }\end{array}$

\section{SPONSORS}

UNIVERSITY OF BRITISH COLUMBIA

UNIVERSITY OF SOUTHERN CALIFORNIA

CALIFORNIA INSTITUTE OF TECHNOLOGY

UNIVERSITY OF CALIFORNIA, BERKELEY

STANFORD RESEARCH INSTITUTE

UNIVERSITY OF CALIFORNIA, DAVIS

STANFORD UNIVERSITY

UNIVERSITY OF CALIFORNIA, LOS ANGELES

WASHINGTON STATE COLLEGE

UNIVERSITY OF CALIFORNIA, SANTA BARBARA

UNIVERSITY OF WASHINGTON

UNIVERSITY OF NEVADA

OREGON STATE COLLEGE

AMERICAN MATHEMATICAL SOCIETY

UNIVERSITY OF OREGON

HUGHES AIRCRAFT COMPANY

Mathematical papers intended for publication in the Pacific Journal of Mathematics should be typewritten (double spaced), and the author should keep a complete copy. Manuscripts may be sent to any of the editors. Manuscripts intended for the outgoing editors should be sent to their successors. All other communications to the editors should be addressed to the managing editor, E.G. Straus, at the University of California Los Angeles 24, California.

50 reprints of each article are furnished free of charge; additional copies may be obtained at cost in multiples of 50 .

The Pacific Journal of Mathematics is published quarterly, in March, June, September, and December. The price per volume (4 numbers) is $\$ 12.00$; single issues, $\$ 3.50$; back numbers (Volumes $1,2,3$ ) are available at $\$ 2.50$ per copy. Special price to individual faculty members of supporting institutions and to individual members of the American Mathematical Society: $\$ 4.00$ per volume; single issues, $\$ 1.25$.

Subscriptions, orders for back numbers, and changes of address should be sent to the publishers, University of California Press, Berkeley 4, California.

Printed at Ann Arbor, Michigan. Entered as second class matter at the Post Office, Berkeley, California.

* To be succeeded in 1955, by H.L. Royden, Stanford University, Stanford, California.

** To be succeeded in 1955, by E.G. Straus, University of California, Los Angeles 24, Calif.

UNIVERSITY OF CALIFORNIA PRESS - BERKELEY AND LOS ANGELES

COPYRIGHT 1954 BY PACIFIC JOURNAL OF MATHEMATICS 


\section{Pacific Journal of Mathematics}

\section{Vol. 4, No. $2 \quad$ June, 1954}

Henry Ludwig Alder, Generalizations of the Rogers-Ramanujan

identities ........................................ 161

E. M. Beelsey, Concerning total differentiability of functions of class $P$. . . 169

L. Carlitz, The number of solutions of some special equations in a finite

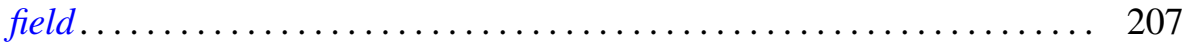

Marshall Hall, On a theorem of Jordan ............................ 219

J. D. Hill, Remarks on the Borel property..................... 227

Joseph Lehner, Note on the Schwarz triangle functions ............... 243

Arthur Eugene Livingston, A generalization of an inequality due to

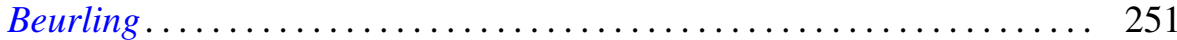

Edgar Reich, An inequality for subordinate analytic functions .......... 259

Dan Robert Scholz, Some minimum problems in the theory of functions ... . 275

J. C. Shepherdson, On two problems of Kurepa ................. 301

Abraham Wald, Congruent imbedding in F-metric spaces ............ 305

Gordon L. Walker, Fermat's theorem for algebras ............... 317 